doi:10.1136/archdischild-2012-302724.0592

OM Horlenko, LY Markovtsiy. Pediatrics and Infectious Diseases, Uzhgorod National University, Medical Faculty, Uzhgorod, Ukraine

Background and Aims Second disbioses of the respiratory play the presentative role and had negatively influence on the result of duration of infectious diseases and assists development of immune disbalance of mucous membranes of respiratory tract.

Methods We investigated 24 children with the Frst Dagnosed Pulmonary Tuberculosis (FDPT) in the age from 1-16 years. Research of microflora of respiratory tracts was conducted by a bacteriologic examination of native material (expectoration).

Results The inspected contingent had Pulmonary form of the first diagnosed tuberculosis. The patients concluded: childs to 3 years $50.00 \%$. other $50.00 \%$ children contained the group of pubertat period. Distributing on the forms of tubercular process: primary tubercular complex $-25.00 \%$, pulmonary focus tuberculose $12.50 \%$, disseminated tuberculosis - $25.00 \%$, infiltrative tuberculosis $37.50 \% .58 .30 \%$ children had assotiatin pathology with the FDPT: anaemia in $25.00 \%$ cases, pneumonia $-8.30 \%$, HIV $-8.30 \%$. In microbiological culture was confirmed presence of $\mathrm{M}$. tuberculosis in $33.30 \%$ cases. The destructive chang in lung $16.70 \%$ cases was identificated. N.sicca was presented in $40.00 \%$ children with the normal microflora and S.epidermidis - in $60.00 \%$. In $50.00 \%$ cases of children with the FDPT disbioses violations was identificated after the beginning of using of antiphthisic treatment. Disbioses as a monoculture found in $83.3 \%$ cases, in $16.70 \%$ cases - as associations of cultures. In $33.30 \%$ cases found out Escherichia coli, in the $16.70 \%$ - K.pneumonia, in the $50.00 \%$ cases - Candida A.

Conclusions On the basis of the conducted researches are set presence of respiratory dysbiosis in children with the FDPT.

\section{PERSISTENTLY ELEVATED RIGHT VENTRICULAR INDEX OF MYOCARDIAL PERFORMANCE IN PRETERM INFANTS WITH INCIPIENT BRONCHOPULMONARY DYSPLASIA}

doi:10.1136/archdischild-2012-302724.0593

C Czernik, S Rhode, B Metze, G Schmalisch, C Buehrer. Klinik für Neonatologie, Charité Universitätsmedizin Berlin, Berlin, Germany

Objectives Elevated pulmonary vascular resistance occurs during the first days after birth in all newborn infants and persists in infants at risk for bronchopulmonary dysplasia (BPD). Pulmonary vascular resistance is higher during the first days after birth and in preterm infants with incipient bronchopulmonary dysplasia (BDP). It is difficult to measure in a non-invasive fashion. We assessed the usefulness of the right ventricular index of myocardial performance (RIMP) to estimate pulmonary vascular resistance in very low birth weight infants.

Study Design Prospective echocardiography on day of life (DOL) $2,7,14$, and 28 in 121 preterm infants (median [quartiles] gestational age 28 [26-29] weeks, birth weight 998 [743-1225] g) of whom 36 developed BPD (oxygen supplementation at 36 postmenstrual weeks)

Results RIMP derived by conventional pulsed Doppler technique was unrelated to heart rate or mean blood pressure. RIMP on DOL 2 was similar in infants who subsequently did (0.39 [0.33-0.55]) and did not develop BPD (0.39 [0.28-0.51], p=0.467). RIMP declined steadily in non-BPD infants but not in BPD infants (DOL 7: $0.31[0.22-0.39]$ vs. $0.35[0.29-0.48], p=0.014$; DOL 14: $0.23[0.17-$ 0.30 ] vs. 0.35 [0.25-0.43], $\mathrm{p}<0.001$; DOL 28: 0.21 [0.15-0.28] vs. 0.31 [0.21-0.35], $\mathrm{p}=0.015)$.

Conclusions In preterm infants, a decline in RIMP after birth was not observed in those with incipient BPD. The pattern of RIMP measured in preterm infants is commensurate with that of pulmonary vascular resistance.

\section{A NEONATAL RAT MODEL OF BRONCHOPULMONARY DYSPLASIA INDUCED BY PRE- AND POSTNATAL INFLAMMATION WITHOUT EXPOSURE TO HYPEROXIA}

doi:10.1136/archdischild-2012-302724.0594

${ }^{1,2} \mathrm{CW}$ Choi, ${ }^{1,3} \mathrm{HJ}$ Lee, ${ }^{1} Y \mathrm{~J}$ Lee, ${ }^{1,2} \mathrm{JM}$ Lee, ${ }^{2} \mathrm{JA}$ Lee, ${ }^{2} \mathrm{EK}$ Kim, ${ }^{2} \mathrm{HS}$ Kim, ${ }^{1,2 \mathrm{BI}}$ Kim, ${ }^{2} \mathrm{JH}$ Choi. ${ }^{1}$ Medical Research Institute, Seoul National University Bundang Hospital, Seongnam; ${ }^{2}$ Department of Pediatrics, Seoul National University College of Medicine; ${ }^{3}$ Department of Pediatrics, Hanyang University Hospital, Seoul, Republic of Korea

Purpose We tested if pre- or postnatal inflammation can develop BPD per se and if there are any interaction between prenatal and postnatal inflammation.

Methods Two days before delivery (E20), $1 \mu \mathrm{g}$ of lipopolysaccharide (LPS) or vehicle (V) was injected into each amniotic sac, and after birth $0.25 \mathrm{mg} / \mathrm{kg}$ of LPS or vehicle was injected into peritoneum on P1, P3, and P5. This led to four experimental groups. On P7 and P14, their lungs and hearts were harvested, and alveolarization and lung vascular density were evaluated.

Results Morphometric analysis of P7 lungs revealed that both preLPS+postLPS group and V+postLPS group had significantly larger and less complex airspaces and small alveolar surface area than $\mathrm{V}+\mathrm{V}$ group. On P14, only V+postLPS group had significantly larger and less complex airspaces than $\mathrm{V}+\mathrm{V}$ group. Howevere, alveolar surface areas were significantly smaller both in preLPS+postLPS group and $\mathrm{V}+$ postLPS group than in $\mathrm{V}+\mathrm{V}$ group. Lung vascular density of both preLPS+postLPS group and V+postLPS group was significantly lesser than $\mathrm{V}+\mathrm{V}$ group.

Conclusions At these intra-amniotic and postnatal systemic LPS doses, prenatal intra-amniotic LPS injection per se did not affect postnatal alveolar and pulmonary vascular development, while postnatal systemic LPS injection significantly inhibited alveolar and pulmonary vascular development regardless of whether prenatal intra-amniotic LPS was injected or not. There was no definite interaction between intra-amniotic LPS and postnatal systemic LPS on the lung development. This rat model of BPD could be used as a valuable tool for testing the effect of anti-inflammatory agents on the prevention of $\mathrm{BPD}$.

\section{RISK FACTORS FOR BRONCHO-PULMONARY DYSPLASIA IN VERY-LOW-GESTATIONAL-AGE INFANTS}

doi:10.1136/archdischild-2012-302724.0595

${ }^{1} \mathrm{~A}$ Valls-i-Soler, ${ }^{2} \mathrm{~A}$ Azpeitia, ${ }^{3} \mathrm{H}$ Hummler, ${ }^{4} \mathrm{H}$ Halliday, European Neonatal Network (EuroNeoNet). 'Cruces University Hospital; ${ }^{2}$ BIOEF, Barakaldo, Spain: ${ }^{3}$ Pediatrics and Adolescent Medicine University Hospital, UIm, Germany; ${ }^{4}$ Institute Clinical Science, Belfast, UK

Background BPD is a severe condition that has decreased in severity but remains a major long-term adverse outcome of surviving Very Low Gestational Age (VLGA) infants.

Aim To determine the BPD rate and evaluate its predictive and associated factors

Methods BPP (need for supplemental $\mathrm{O}_{2}$ at $36 \mathrm{wks}$ CGA) rate and associated risk factors were analysed in a cohort of 24,087 VLGA infants admitted from 2006 to 2010 to 174 EuroNeoNet NICUs. Non-parametric independent tests and logistic regression models were performed to predict BPD, using crude and adjusted odd ratios (OR) to determine perinatal and early neonatal associations. Predictive capacity was assessed by Hosmer-Lemeshow test and discrimination by area under ROC curve (AUC).

Results BPD was diagnosed in 16\% (95\%CI: $(15.4 \%-16.1 \%)$ ) of infants, who had significantly lower GA, BW and Apgar scores. They were more frequently male, from single pregnancies, more often had 